\title{
APROPIACIÓN TECNOLÓGICA COMO EJE EN EL DISEÑO DE UNA EXPERIENCIA EDUCATIVA EN LA LICENCIATURA DE ENSEÑANZA DE LOS ESTUDIOS SOCIALES
}

\section{TECHNOLOGICAL APPROPRIATION AS THE AXIS IN THE DESIGN OF A LEARNING EXPERIENCE IN THE SOCIAL STUDIES MAJOR DEGREE}

Fecha de recepción: 17/10/2017

Fecha de aceptación: 06/11/2017

Resumen: Las herramientas tecnológicas ofrecen al docente una amplia gama de opciones para dinamizar los procesos de aprendizaje. Sin embargo, la implementación de tecnologías en la enseñanza per se no garantiza aprendizajes profundos y dinámicos. Es decir, el uso de tecnologías requiere de un proceso de diseño pedagógico pertinente, en el cual se determine el cómo y el cuándo incorporar las tecnologías en el proceso de enseñanza, así como el tipo de aprendizajes que se espera lograr. El presente documento reflexiona sobre una experiencia educativa del autor en la licenciatura en la enseñanza de los Estudios Sociales y Educación Cívica, con el objetivo de establecer un modelo de tecnologías para el aprendizaje. La propuesta plantea dos fases de implementación que incluyen la identificación y caracterización de las distintas herramientas tecnológicas según sus cualidades educativas, el diseño de las actividades de mediación y la implementación en clase y a distancia. De tal manera, desde la propuesta del modelo de implementación mostrado, se pretende reflexionar sobre los retos y las oportunidades del uso de tecnologías para generar aprendizajes pertinentes, críticos y creativos, según el grado de apropiación tecnológica tanto del docente como del estudiantado.

Palabras clave: apropiación tecnológica; herramientas educativas; aprendizaje socio-cultural; e-learning; estudios sociales; educación cívica.

\begin{abstract}
Technological tools offer the teacher a wide range of options to streamline learning processes. However, the implementation of technologies in education per se, do not guarantee deep and dynamic learning. That is, the use of technologies requires a process of relevant pedagogical design, which determines how and when to incorporate technologies into the teaching process, as well as the type of learning that is expected to be achieved. The present paper systematizes some educational experiences with the objective of establishing a model of implementation of technologies for learning. The model proposes phases of implementation that include the identification and characterization of the different technological tools according to their educational qualities, design of the mediation activities and their implementation in class. Therefore it is intended to reflect on the challenges and opportunities of the use of technologies to generate relevant, critical and creative learning, according to the degree of technological appropriation of both teacher and student.
\end{abstract}

Keywords: technological appropriation; technologies; socio-cultural learning; e-learning; social studies; civic education.

\footnotetext{
* Costarricense. Licenciado en la Enseñanza de los Estudios Sociales y la Educación Cívica por la Universidad Nacional (UNA). Actualmente, se desempeña como docente en la Escuela de Historia de esa misma universidad. Correo electrónico: degs05@gmail.com
} 


\section{Introducción}

Las tecnologías de la información han modificado sustancialmente las nociones tradicionales del conocimiento, de la información y del aprendizaje. Un rasgo determinante es la inmediatez con la cual se obtiene información, así como la infinidad de fuentes de información disponible en la web. La brecha digital parece cada vez más determinada más por el saber usar que por el acceso, pues se puede tener cierto acceso a tecnologías básicas como un computador o teléfono móvil inteligente, no obstante la forma de emplearlo marca la diferencia. El acceso a las tecnologías de la información y comunicación requiere cada vez más de formas adecuadas de uso e implementación para resolver problemas cotidianos. En el ámbito educativo, el impacto de las tecnologías evidencia la necesidad de redefinir la forma como se conciben los procesos de enseñanza y, por ende, de aprendizaje en el marco de la sociedad de la información y el conocimiento. Distinguir información de conocimiento permite separar procesos pedagógicos entre aquellos que fomentan solamente la acumulación de datos, de aquellas actividades educativas diseñadas para promover construcción de pensamiento original. En este sentido, 2 actualizar la enseñanza al contexto informatizado y digital de hoy presenta una serie de retos para docentes y estudiantes de la educación superior. Las herramientas tecnológicas ofrecen al docente una amplia gama de opciones para dinamizar los procesos de aprendizaje. Sin embargo, la implementación de tecnologías en la enseñanza per se no garantizan aprendizajes profundos y dinámicos. Es decir, el uso de tecnologías requiere de un diseño pedagógico pertinente, en el cual se determine el cómo y el cuándo incorporar las tecnologías en el proceso de enseñanza, así como el tipo de aprendizajes que se espera lograr. De manera que, en esta ocasión quisiera compartir algunas experiencias educativas que he tenido en los cursos del bachillerato y licenciatura en la enseñanza de los Estudios Sociales, con el objetivo de explorar algunos elementos que permitan establecer un modelo de implementación de tecnologías para el aprendizaje. La propuesta plantea fases de implementación que incluyen la identificación y caracterización de las distintas herramientas tecnológicas según sus cualidades educativas, el diseño de las actividades de mediación y su implementación en clase y a distancia. No sin antes 
presentar algunas nociones conceptuales que dan soporte teórico-pedagógico a las experiencias, para finalmente reflexionar sobre los retos y las oportunidades del uso de tecnologías para generar aprendizajes pertinentes, críticos y creativos, según el grado de apropiación tecnológica tanto del docente como del estudiantado.

\section{¿Para qué implementar herramientas tecnológicas en la enseñanza? Cuestiones teórico- conceptuales de la experiencia}

Vivimos en la era de la información, nada ni nadie cuestiona ese hecho. No obstante ¿qué significa tal afirmación? Tal vez el referente más conocido en este tema sea Manuel Castells, quien en primer lugar indica que la sociedad de la información no es lo mismo que sociedad informacional. Para Castells, "el término informacional indica el atributo de una forma específica de organización social en la que la generación, el procesamiento y la transmisión de información se convierten en las fuentes fundamentales de la productividad y el poder, debido a las nuevas condiciones tecnológicas que surgen en este período histórico" (Castells, 1998: 47.) El acento está, siguiendo la reflexión de Castells, no en la concepción que da importancia a la información per se, como si fuese un producto absoluto y neutral que le es dado a la sociedad, más bien en la incidencia que ejerce la producción del conocimiento, la estructuración, los mecanismos y las redes de flujo de la información que de él se desprende. Debemos preguntarnos entonces ¿quiénes producen el conocimiento, lo seleccionan y procesan para que sea considerado información válida? El hecho de que la información posea todas esas connotaciones obliga a establecer procesos de jerarquización donde las cuotas de poder las distribuyen los dueños del capital en alianza con los gobiernos y productores de bienes culturales, en el que el capitalismo se revoluciona así mismo posibilitando la acumulación permanente legitimada por una sociedad que asume como natural tal ciclo productivo del conocimiento. Castells precisa ese carácter al señalar que:

Lo que caracteriza a la revolución tecnológica actual no es el carácter central del conocimiento y la información, sino la aplicación de ese conocimiento e información a aparatos de generación de conocimiento y procesamiento de la 
información/comunicación, en un círculo de retroalimentación acumulativo entre la innovación y sus usos (1998: 58).

Podemos colegir de lo anterior que las personas en general, docentes y estudiantes en particular, se enfrentan al reto de convertirse en productores de conocimientos mediante el empleo y uso razonado de las tecnologías o ser consumidores de información, empleando las tecnologías solamente como medios sustitutivos de información. Entiéndase medios sustitutivos como aquella situación en la que se cambia un soporte tradicional de información por uno digital, por ejemplo los medios escritos físicos por redes sociales. La implementación de herramientas tecnológicas desde mi experiencia parte de la idea de desarrollar niveles de apropiación tecnológica. Entiendo como apropiación tecnológica la relación que se establece entre las personas y las herramientas tecnológicas; relación que requiere del desarrollo de la capacidad para interiorizar, comprender y emplear una herramienta tecnológica en la resolución de problemas cotidianos (Colás y Jiménez, 2008; Fernández y McAnally, 2015). Ahora bien, la apropiación está asociada con la idea vigotskiana (1978) de que el aprendizaje se basa en el uso de herramientas o dispositivos culturales cuya apropiación se “...realiza en contextos donde los sujetos mantienen una intersubjetividad y se hace efectiva cuando el individuo es capaz de utilizar cualquier recurso tecnológico en cualquier actividad cotidiana y en contextos distintos al que asoció su dominio" (Wertsch, 1988; Fernández y McAnally, 2015).

\section{¿Cómo y cuándo implementar? Precisiones de diseño homogeneizar subtítulos}

Aclarado el concepto y su base epistémica. Es importante considerar que la apropiación tecnológica es un proceso, por lo tanto posee niveles, grados de apropiación, tanto para el docente como para estudiantes. En el caso docente, el grado de apropiación tecnológica determina el impacto del aprendizaje con tecnologías. El docente puede utilizar herramientas tecnológicas como sustitución de dispositivos tradicionales: cambiar la pizarra por presentaciones Power Point o Prezi, evaluar mediante formularios en línea, por ejemplo. Puede llegar al nivel de producir sus propios recursos didácticos con algunas herramientas digitales. Sin embargo, estos niveles solo muestran que la tecnología se utiliza de forma cotidiana, más no 
necesariamente apunta a generar procesos de construcción paralela con respecto al estudiantado, porque no cambia la forma de mediación: transmisión de información. En el esquema 1, se visualizan cuatro niveles de apropiación docente, aclaro, no son los únicos niveles, sino aquellos básicos que, siguiendo a Celaya, Lozano y Ramírez (2010) pueden ser puntos de referencia.

\section{Esquema 1}

Niveles de apropiación tecnológica docente

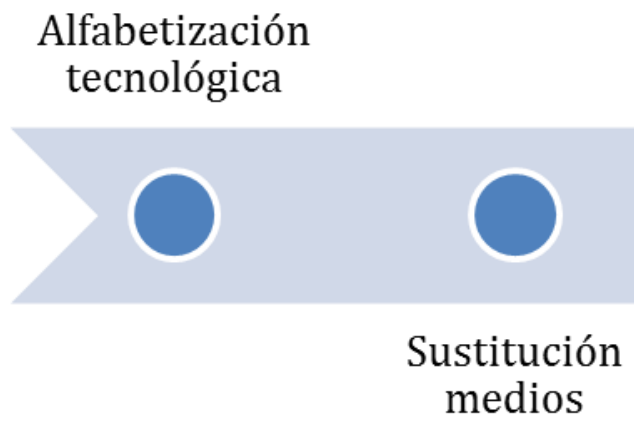

Fuente: elaboración propia con base en Celaya, Lozano y Ramírez (2010).
Producción

didáctica 


\section{Esquema 2 \\ Niveles de apropiación tecnológica estudiantes}

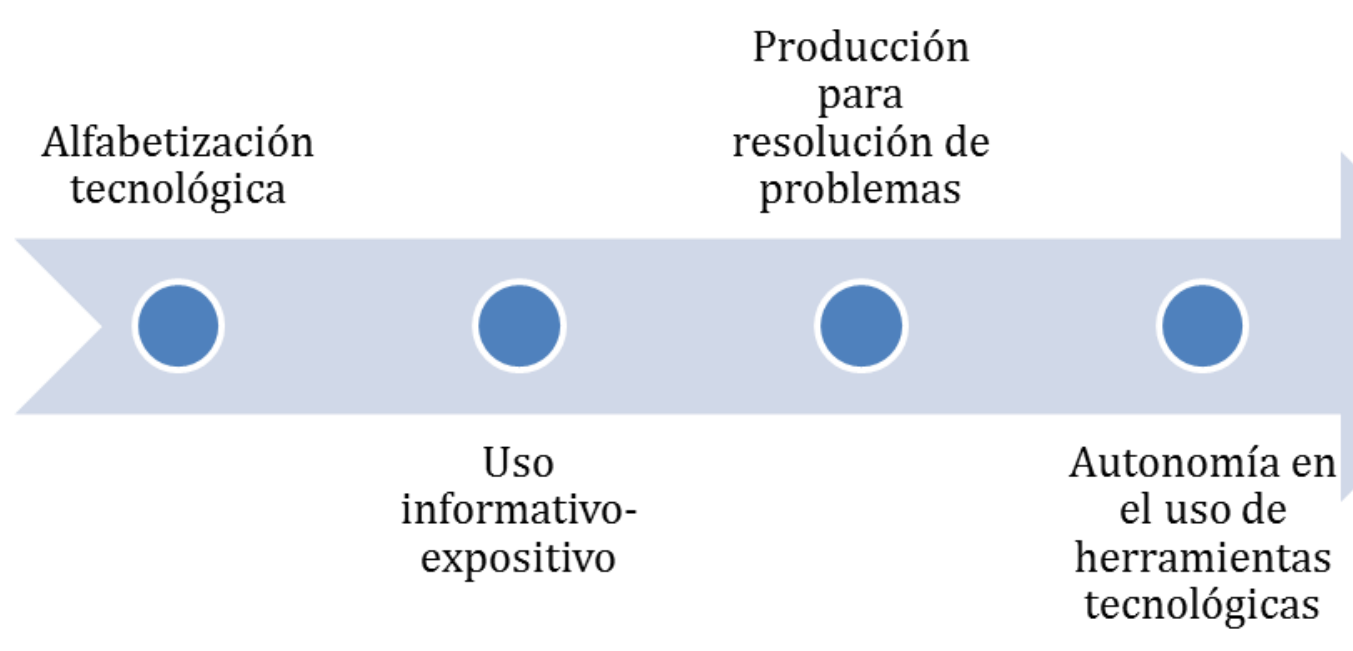

Fuente: elaboración propia.

Ahora bien, en cuanto a las herramientas tecnológicas, para el caso de la experiencia descrita aquí, entiéndase aquellos programas, software o aplicaciones que permiten el uso en procesos educativos, las cuales pueden catalogarse según su función, uso y finalidad; además se debe considerar el acceso -libre o de pago-, la confiabilidad y usabilidad. Por cuestiones de espacio, me limito a presentar el siguiente cuadro resumen que ilustra los criterios y las herramientas utilizadas en la experiencia tratada en este escrito. 


\section{Cuadro 1}

Tipos de herramientas tecnológicas, criterios de selección y características

\begin{tabular}{|l|l|l|}
\hline \multicolumn{1}{|c|}{ Tipo } & \multicolumn{1}{|c|}{ Herramientas } & \multicolumn{1}{c|}{ Criterio } \\
\hline $\begin{array}{l}\text { Ofimática: aplicaciones de } \\
\text { uso frecuente como } \\
\text { procesador de texto, gráficos } \\
\text { y datos. }\end{array}$ & $\begin{array}{l}\text { Word, Excel, Power } \\
\text { Point, Publisher. }\end{array}$ & $\begin{array}{l}\text { - Uso común } \\
\text { - Accesible y portable } \\
\text { - Confiable }\end{array}$ \\
\hline $\begin{array}{l}\text { Web/en línea: aplicaciones o } \\
\text { herramientas de acceso libre } \\
\text { disponibles en la web. }\end{array}$ & $\begin{array}{l}\text { Pick to Chart, Lino It, } \\
\text { Google Drive, } \\
\text { Powtoon. }\end{array}$ & $\begin{array}{l}\text { - Confiable } \\
\text { - Acceso libre }\end{array}$ \\
\hline $\begin{array}{l}\text { Producción: herramientas o } \\
\text { aplicaciones de acceso libre } \\
\text { que posibilitan crear diversos } \\
\text { objetos de aprendizaje. }\end{array}$ & $\begin{array}{l}\text { QR Code, Freemind, } \\
\text { Cmapstool, } \\
\text { Atubecatcher }\end{array}$ & $\begin{array}{l}\text { - Acceso libre } \\
\text { - Portable }\end{array}$ \\
\hline
\end{tabular}

Fuente: elaboración propia.

Las herramientas presentadas en el cuadro 1 se inscriben en un espacio que sirve como punto de encuentro entre docente, estudiantes, herramientas e información: el aula virtual UNA. El aula virtual es un ambiente digital en el cual confluyen varios elementos que permiten la mediación educativa virtual, asincrónica y colaborativa. En nuestra universidad el soporte del ambiente virtual es moodle 3.1. De esta manera, la experiencia se estructura desde la siguiente manera: espacio físico -sesiones presenciales-, aula virtual UNA -sesiones virtuales paralelas-, herramientas utilizadas por el docente para los procesos de enseñanza y herramientas presentadas para trabajo -aprendizaje- en el aula virtual. 


\section{Esquema 3 \\ Dinámica enseñanza-aprendizaje}

Fuente: elaboración propia.

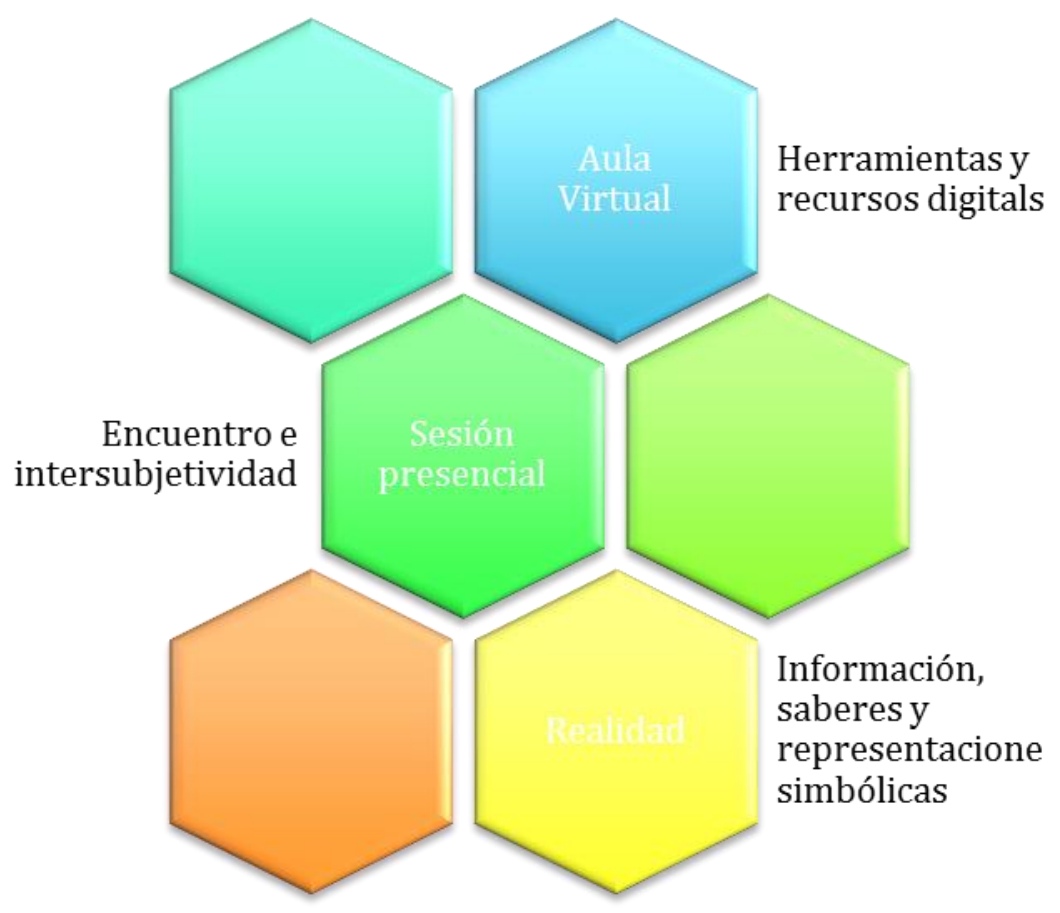

\section{Fases}

A continuación se sintetizan, a manera de pasos, el modelo de implementación de herramientas tecnológicas en la dinámica de enseñanza en dos cursos distintos: uno de tipo regular, de grado con un grupo de segundo año del bachillerato en enseñanza de los Estudios Sociales, el otro en la licenciatura de la misma carrera, el cual es de tipo bimodal y se enfoca en el desarrollo de una investigación socio-educativa. La primera fase corresponde a la preparación del curso, el planeamiento y la selección de las estrategias de mediación y herramientas a utilizar. Esta fase comprende, a saber:

1. Objetivos del curso y aprendizajes específicos: Los establecidos según el plan de la carrera, además de aquellos que no se contemplaron pero se consideran para actualizar el curso. De ahí, se determinan los aprendizajes más importantes sobre los cuales se plantea la mediación. 
2. Curaduría de herramientas según aprendizajes a lograr: previo conocimiento de varias herramientas, tomando en cuenta la facilidad de acceso, uso y si es para uso de los estudiantes o del docente. Las herramientas utilizadas por el docente son aquellas en las cuales construye, elabora y presenta su material didáctico. Para los estudiantes, son las herramientas que aprenderán a utilizar en clase para una actividad específica, sea para presentar información o para crear productos digitales, como revistas, audios, códigos $\mathrm{QR}$, entre otros. La selección de las herramientas parten del objetivo pedagógico, la habilidad requerida para el aprendizaje según el contenido y la utilidad del producto a crear.

3. Secuenciación virtual-presencial: al tratarse de un curso bimodal, el trabajo asincrónico requiere una secuenciación con las actividades presenciales, pues de ello depende que el proceso no se pierda durante el trabajo a distancia. Las sesiones presenciales tienen como objetivo delimitar el objeto de estudio -contenido u otro-, concretar la intersubjetividad al someter las reflexiones a valoración del grupo utilizando soportes como presentaciones o videos elaborados durante el trabajo a distancia. Las sesiones virtuales permiten distribuir el trabajo a distancia para completar tareas específicas, cuyo resultado debe evidenciarse en un foro o producto específico -documento, presentación o animación, etc. -. Cuando se trate de introducir herramientas que los estudiantes no conocen la sesión presencial permite, mediante la estrategia del taller, desarrollar la temática al tiempo que se implementa la herramienta. La misma será mejorada en la actividad virtual.

En la que he denominado segunda fase, se considera ya el momento en que se desarrolla el trabajo educativo, sin ser un proceso separado de la primera fase. Esta comprende:

4. Introducción de herramientas: cuando la actividad planteada presenta el uso de una herramienta, la selección es a priori y debe ser de fácil manipulación, de acceso libre y ser coherente con el objetivo educativo. Las sesiones presenciales se plantean, en su primera parte como taller de trabajo con la herramienta. Aquí se explora la misma de manera general; si requiere descarga o si es en línea, se accede e inicia la implementación 
de la misma a modo de inducción. El taller utiliza el contenido correspondiente para la sesión, la herramienta es solo el medio mediante el cual se podrá concretar el objetivo pedagógico. La herramienta se continuará utilizando en las sesiones a distancia de forma secuencial para crear un producto que será socializado en el aula virtual y luego en la sesión presencial.

5. Evaluación: No es la evaluación de los productos en términos sumativos, sino específicamente formativos. Se evalúa el alcance de la herramienta en contraste con el objetivo planteado. El producto -en los casos de elaboración de algún producto educativo- permite medir el grado de profundidad en el manejo de la información, de apropiación tecnológica y de reflexión -producción de subjetividad- en torno al objetivo pedagógico.

\section{Implementación del modelo}

A continuación se detalla mediante una matriz el modelo de implementación de algunas herramientas tecnológicas durante el curso de Investigación socio-educativa aplicada a los Estudios Sociales y Educación Cívica. Este curso, corresponde a la licenciatura en la enseñanza de dichas asignaturas y se imparte de manera bimodal utilizando la plataforma UNA aula virtual moodle.

\section{Cuadro 2}

\begin{tabular}{|c|c|c|c|}
\hline Objetivo pedagógico & Habilidad & $\begin{array}{c}\text { Herramientas } \\
\text { tecnológicas }\end{array}$ & Actividades \\
\hline $\begin{array}{l}\text { Explorar problemas de } \\
\text { investigación socio- } \\
\text { educativa en el área de } \\
\text { ES y EC. }\end{array}$ & $\begin{array}{ll}\text { - } & \text { Problematizar la } \\
& \text { realidad. } \\
\text { - } & \text { Análisis de } \\
\text { contextos. } \\
\text { - Organización de } \\
\text { información. } \\
\text { - Inducción. }\end{array}$ & $\begin{array}{l}\text {-Aula virtual } \\
\text {-Código QR: lecturas. } \\
\text {-Lino It } \\
\text {-Google Drive }\end{array}$ & $\begin{array}{l}\text { Foro de } \\
\text { acercamiento a } \\
\text { problemas socio- } \\
\text { educativos. } \\
>\begin{array}{l}\text { Lluvia de ideas y } \\
\text { clasificación }\end{array} \\
\text { intersubjetiva de } \\
\text { problemas socio- } \\
\text { educativos } \\
\text { mediante la pizarra } \\
\text { online Lino It. } \\
\text { Documento grupal } \\
\text { asincrónico de }\end{array}$ \\
\hline
\end{tabular}




\begin{tabular}{|c|c|c|c|}
\hline & & & $\begin{array}{l}\text { problemas en } \\
\text { Google Drive. } \\
\text { Taller códigos QR: } \\
\text { utilidad y uso. }\end{array}$ \\
\hline $\begin{array}{l}\text { Diseñar un objeto de } \\
\text { estudio de } \\
\text { investigación socio- } \\
\text { educativa }\end{array}$ & $\begin{array}{ll}\text { - } & \text { Análisis } \\
\text { información. } \\
\text { - } & \text { Inferencia } \\
& \text { situaciones socio- } \\
& \text { educativas. } \\
\text { - } & \text { Organización de } \\
\text { procesos. }\end{array}$ & $\begin{array}{l}\text {-Aula virtual } \\
\text {-Picktochart } \\
\text {-Google drive }\end{array}$ & $\begin{array}{l}\text { Foro discusión por } \\
\text { subgrupos. } \\
\text { Taller elaboración } \\
\text { infografías con } \\
\text { Picktochart: } \\
\text { aplicada al } \\
\text { diagnóstico para } \\
\text { detección de un } \\
\text { posible objeto de } \\
\text { estudio. } \\
\text { Organización y } \\
\text { sistematización del } \\
\text { proceso de } \\
\text { investigación en } \\
\text { documento } \\
\text { colaborativo en } \\
\text { Google Drive. }\end{array}$ \\
\hline $\begin{array}{l}\text { Comprender las } \\
\text { nociones teórico- } \\
\text { conceptuales de la } \\
\text { metodología de la } \\
\text { investigación socio- } \\
\text { educativa. }\end{array}$ & $\begin{array}{l}\text { Inferencia. } \\
\text { - Análisis de } \\
\text { fuentes } \\
\text { bibliográficas. } \\
\text { - Abstracción } \\
\text { conceptual. }\end{array}$ & $\begin{array}{l}\text {-Power Point (mix) } \\
\text {-Excel } \\
\text {-Powtoon } \\
\text {-Google Drive } \\
\text {-Emaze }\end{array}$ & $\begin{array}{ll} & \text { Taller Powtoon. } \\
& \text { Taller matrices en } \\
& \text { Excel. } \\
& \text { Taller de diseño } \\
& \text { reportes Emaze. } \\
> & \text { Elaboración de } \\
\text { contenidos vía } \\
\text { Google Drive para } \\
\text { implementar cada } \\
\text { herramienta, a } \\
\text { partir de preguntas } \\
\text { generadoras sobre } \\
\text { los tres conceptos: } \\
\text { paradigma, } \\
\text { enfoque y tipo de } \\
\text { investigación. } \\
\text { Socialización de } \\
\text { productos en } \\
\text { actividad virtual. }\end{array}$ \\
\hline
\end{tabular}

Fuente: elaboración propia.

Se observa en el cuadro que hay relación horizontal entre el objetivo y las habilidades a potenciar en la dinámica de enseñanza. Los tres objetivos reflejan los primeros acercamientos en la investigación socio-educativa, por lo que las actividades propuestas persiguen que cada 
estudiante piense sobre la educación como proceso social, dinámico y cambiante, el cual presenta problemas particulares para la enseñanza de los Estudios Sociales y la Educación Cívica. La capacidad para comprender la realidad y problematizarla a partir de situaciones requiere claridad conceptual sobre la producción de conocimientos. La investigación socioeducativa es una herramienta para convertir los problemas en objetos de estudio relevantes, pertinentes y viables de generar nuevo conocimiento. Investigar es por ello una actividad de aprendizaje en tanto supone pensar sistemáticamente la totalidad de un fenómeno y en sus ramificaciones. Los tres objetivos atienden el andamiaje para ese pensar sistemático que es una habilidad socio-cognitiva al mismo tiempo. Subyacente a tal proceso, la propuesta busca darle soportes tecnológicos a las actividades para catalizar las habilidades, tales como indagar, analizar, sintetizar, organizar, seleccionar y clasificar. Así mismo, abstraer conceptos teóricos específicos al campo de la investigación -paradigma, enfoque, método-; siempre desde el aprender haciendo y el trabajo colaborativo virtual y presencial. La introducción de herramientas desconocidas se logra mediante talleres donde se trabajaron conceptos, procesos y lo relacionado con la temática de la investigación. Con respecto a las herramientas ya conocidas por el estudiantado, como las que comprenden el paquete ofimático, se amplió su uso en funciones poco utilizadas como la generación de bibliografía, la creación de matrices analíticas y en especial, su uso en el ambiente virtual Google Drive. En ello el trabajo colaborativo se profundiza porque se evidencia el proceso de construcción de ideas que se van modificando según cada estudiante avanza con sus aprendizajes. El docente puede observar la construcción, organización, elaboración de los informes que sirven como base para el futuro diseño del anteproyecto de investigación. Las demás herramientas que permiten sintetizar la información para comunicarla en las sesiones de realimentación contribuyen en las habilidades de selección, inferencia y categorización de información según el objetivo de la comunicación.

\section{A modo de conclusión}

Las herramientas tecnológicas educativas ni cualquier otro soporte digital sustituyen la labor profesional del docente que consiste en crear las condiciones adecuadas para generar situaciones de aprendizaje. La condición hiperinformatizada de la sociedad actual, urge más que 
nunca de habilidades de pensamiento para seleccionar, descartar y contrastar aquello que parece verdadero pero no lo es. Aprender a discernir es poner en movimiento el intelecto para pensar sistemáticamente, empleando las herramientas cognitivas mejor desarrolladas y con ello desarrollar la capacidad subjetiva desde la cual cada estudiante asume como sujeto activo de la sociedad y no ente pasivo. Apropiarse de la tecnología para aprender y aprender profundamente y críticamente, humaniza y evita la cosificación por un sistema. Las herramientas tecnológicas actualizan esas formas de acercarnos a la información, al manejo de los datos y las verdades. Su implementación es una actualización del lenguaje como herramienta para el mundo tecnológico. Las herramientas deben y pueden profundizar habilidades, nunca sustituir las capacidades transfiriendo las funciones del pensar a actividades de automatización. Los estudiantes se empoderan de su proceso de aprendizaje en tanto tienen la convicción de que están aprendiendo, y cómo aprenden. La posibilidad metacognitiva del aprendizaje con herramientas tecnológicas, intrínsecamente está determinado por la capacidad docente para aprender los lenguajes contemporáneos de la enseñanza con tecnologías cuya función estriba en potenciar aprendizajes complejos y profundos. En cuanto a los retos, la actualización constante es uno de los mayores, ya que día a día surgen nuevas aplicaciones y herramientas por conocer, probar y experimentar para que puedan llegar al aula sin que se conviertan en obstáculos del aprendizaje. El docente se auto-capacita y crea una base de datos que puede ir mejorando en tanto pueda reflexionar sobre su propia práctica docente. Otro reto encontrado es la resistencia que en algunos casos muestran estudiantes, ya que por una cultura escolarizada que aún cargan, dan prioridad al contenido y no a los procesos. Al utilizar herramientas, el docente debe enseñar sus usos, características y posibilidades, de lo contrario el estudiante no podrá comprender el objetivo y vínculos entre el contenido y la finalidad de utilizar las herramientas.

Finalmente, tres razones resumen las oportunidades de la implementación de tecnologías, a saber: 1) Contextualizar la dinámica enseñanza-aprendizaje: esto es, reconocer que el mundo digital ofrece infinidad de recursos, información y nuevas formas de interactuar y conocer, a los que no podemos obviar pero requieren un abordaje previo para que sean pertinentes con nuestros objetivos pedagógicos y contenidos temáticos; 2) Dinamizar los procesos intersubjetivos combinando espacios de aprendizaje físico y virtual: El aula como espacio de socialización debe 
trascender hacia otros espacios, incorporar otras voces que permitan a docente y estudiantes la experiencia de aprendizajes colectivos. Los ambientes virtuales ofrecen la posibilidad de interacciones constantes y diversas. La combinación de ambos espacios en la secuenciación de los aprendizajes contribuye al desarrollo una dinámica intersubjetiva que decanta aprendizajes y habilidades en medio del intercambio con los demás, con otras fuentes, con otros medios, reforzando la idea de comunidad de aprendizaje al tiempo que expande la visión de la clase al mundo y; 3) asumir el aprendizaje como la construcción propia del sujeto: si cada estudiante logra apropiarse de las herramientas -cognitivas, tecnológicas, sociales- para asumir su aprendizaje como una forma de construirse a sí mismo, estamos promoviendo la autonomía, la metacognición, el aprendizaje como algo dinámico y no como la transmisión de información. Aprender, saber cómo se aprende y crear formas pensar, contribuyen a un sujeto social más crítico, más comprometido con sigo mismo y con los demás.

\section{Bibliografía}

Castells, M. (1998). La era de la información. Vol. 1 La sociedad red. Madrid, España: Alianza.

Celaya, R., Lozano, F., y Ramírez a, M. (2010). Apropiación tecnológica en profesores que incorporan recursos educativos abiertos en educación media superior. Revista mexicana de investigación educativa, 15(45), 487-513. Recuperado de http://www.scielo.org.mx/scielo.php?script=sci arttext\&pid=S1405$\underline{66662010000200007 \& \operatorname{lng}=\text { es\&tlng=es }}$

Colás, P., Rodríguez, M., y Jiménez, R. (2005). "Evaluación de e-learning. Indicadores de calidad desde el enfoque sociocultural". Teoría de la Educación. Educación y Cultura en la Sociedad de la Información, 6(2). Recuperado de http://www.redalyc.org/articulo.oa?id=201021055003.

Martínez, M. (1999). "El enfoque sociocultural en el estudio del desarrollo y la educación". Revista Electrónica de Investigación Educativa, 1(1). Recuperado de http://redie.uabc.mx/index.php/redie/article/view/6/9

Mejía, M. (2013). La investigación como estrategia pedagógica, una propuesta desde el sur. Bogotá: Ediciones desde Abajo. 
McAnally-Salas, L. Navarro, M. y Rodríguez J. (2006). La integración de la tecnología educativa como alternativa para ampliar la cobertura en la educación superior. Revista Mexicana de Investigación Educativa, 11 (28), pp. 11-30.

Vygotsky, L. (1978). Mind in society: The development of higher psychological processes. Cambridge: Harvard University Press.

Wertsch, J. (1988). Vygotsky y la formación social de la mente. Barcelona, España: Ediciones Paidós Ibérica. 